\title{
Cognitive Correlates of White Matter Growth and Stress Hormones in Female Squirrel Monkey Adults
}

\author{
David M. Lyons, Chou Yang, Stephan Eliez, Allan L. Reiss, and Alan F. Schatzberg \\ Department of Psychiatry and Behavioral Sciences, Stanford University School of Medicine, Stanford, California 94305-5485
}

\begin{abstract}
Neurobiological studies of stress and cognitive aging seldom consider white matter despite indications that complex brain processes depend on networks and white matter interconnections. Frontal and temporal lobe white matter volumes increase throughout midlife adulthood in humans, and this aspect of aging is thought to enhance distributed brain functions. Here, we examine spatial learning and memory, neuroendocrine responses to psychological stress, and regional volumes of gray and white matter determined by magnetic resonance imaging in 31 female squirrel monkeys between the ages of 5 and 17 years. This period of lifespan development corresponds to the years 18-60 in humans. Older adults responded to stress with greater increases in plasma levels of adrenocorticotropic hormone and modest reductions in glucocorticoid feedback sensitivity relative to young adults. Learning and memory did not differ with age during the initial cognitive test sessions, but older adults more often failed to inhibit the initial learned response after subsequent spatial reversals. Impaired cognitive response inhibition correlated with the expansion of white matter volume statistically controlling for age, stress hormones, gray matter, and CSF volumes. These results indicate that instead of enhancing cognitive control during midlife adulthood, white matter volume expansion contributes to aspects of cognitive decline. Cellular and molecular research combined with brain imaging is needed to determine the basis of white matter growth in adults, elucidate its functions during lifespan development, and provide potential new targets for therapies aimed at maintaining in humans cognitive vitality with aging.
\end{abstract}

Key words: brain aging; myelination; cognition; HPA-axis; primates; magnetic resonance (MR) imaging (MRI)

\section{Introduction}

Neurobiological studies of stress, cognitive performance, and aging in humans often focus on the hippocampus and adjacent brain regions (Lupien and Lepage, 2001; Miller and O'Callaghan, 2003). The hippocampus is involved in learning and memory (Eichenbaum, 2000), inhibits the hypothalamic-pituitaryadrenal (HPA) hormone stress response (Herman et al., 2003), and various aspects of hippocampal morphology are altered by stress and aging in rats (McEwen, 1999; Sapolsky, 1999; Lupien and Lepage, 2001; Miller and O'Callaghan, 2003). Diminished hippocampal volumes determined in elderly humans by magnetic resonance imaging (MRI) are taken as evidence that stress during aging contributes significantly to hippocampal atrophy and impairs hippocampal-dependent memory (Lupien et al., 1998).

Far fewer studies have examined the role of white matter growth during midlife adulthood, despite ample evidence that cognitive functions and stress-related brain processes depend on broadly distributed networks and white matter interconnections

Received Nov. 26, 2003; revised Feb. 23, 2004; accepted Feb. 23, 2004.

This work was supported by a Stanford University Medical School Scholars Fellowship and United States Public Health Service Grants MH47573 and MH50604. W. Baumgardner, C. Buckmaster, S. Fryer, S. Kessler, J. Kraemer, K. Parker, A. Sawyer-Glover, E. Schmitt, and J. Zeitzer provided invaluable assistance with various aspects of this research.

Correspondence should be addressed to David M. Lyons, Psychiatry Neuroscience, 1201 Welch Road Medical School Laboratory Surge building, Room P104, Mail Code 5485, Stanford University School of Medicine, Stanford, CA 94305-5485. E-mail: dmlyons@stanford.edu.

D0I:10.1523/JNEUROSCI.0324-04.2004

Copyright $\odot 2004$ Society for Neuroscience $\quad 0270-6474 / 04 / 243655-08 \$ 15.00 / 0$
(Mesulam, 1998; Greenwood, 2000; Kumar and Cook, 2002; Herman et al., 2003). Hippocampal and gray matter volumes decline gradually with aging in adults, whereas frontal and temporal lobe white matter volumes continue to increase in humans throughout the first five decades of life (Courchesne et al., 2000; Bartzokis et al., 2001; Jernigan and Fennema-Notestine, 2004). Adult white matter growth determined in vivo by MRI concurs with the classic postmortem studies of Yakovlev and Lecours (1967).

White matter is composed of axons sheathed in myelin produced by oligodendrocytes. Myelination increases nerve conduction velocities (Baumann and Pham-Dinh, 2001) and facilitates synchronous firing of neurons by reducing the effects of travel distance variability in distributed networks (Salami et al., 2003). Frontal and temporal lobe white matter growth has therefore been viewed as enhancing in humans neuropsychological functions that rely on distributed networks (Greenwood, 2000; Bartzokis et al., 2001; Mulhern et al., 2001; Giedd, 2003). This hypothesis conflicts with evidence from monkeys that myelin production (Peters et al., 2001; Peters and Sethares, 2003) and proliferation of oligodendrocytes (Peters, 1996) in adulthood coincide with increased accumulation of myelin decompaction defects that correlate with cognitive decline (Peters and Sethares, 2002). Stress hyper-reactivity (Seeman et al., 2001) and diminished glucocorticoid feedback (Wilkinson et al., 2001) are additional aspects of aging that conflict with the view that white matter expansion improves distributed brain functions.

Here, we examine relationships between age-related differences in learning and memory, neuroendocrine responses to psy- 


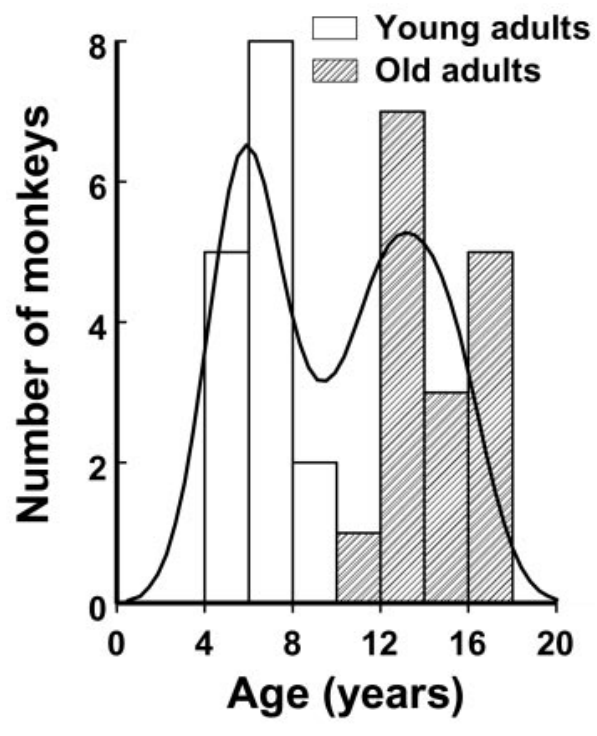

Figure 1. Bimodal age distribution of 31 adult female squirrel monkeys.

chological stress, and volumes of gray and white matter determined in vivo by MRI in 31 female squirrel monkeys between 5 and 17 years of age. This period of lifespan development corresponds to the years 18-60 in humans. Age-related brain changes in monkeys during this time are not confounded by Alzheimer's disease, because this pathology does not occur in monkeys (Walker et al., 1990; Peters et al., 1996). Rodent models are limited because prefrontal connections differ in rats compared with humans and monkeys (Preuss, 1995). Certain features of aging in humans cannot be modeled directly in monkeys, but comparative studies of homologous brain regions in monkeys are essential for understanding the biology of healthy brain aging, stress, and cognition during midlife adulthood in humans.

\section{Materials and Methods}

Subjects. Female squirrel monkeys (Saimiri sciureus) of Guyanese origin that were born and raised at Stanford University (Stanford, CA) served as subjects for the study. From a colony comprised of 57 healthy adult females with a median age of 10 years (range, $4-17$ years of age), matched samples were randomly selected from the young $(<10$ years of age $)$ and older ( $>10$ years of age) age classes. As depicted in the bimodal age distribution in Figure 1, 15 monkeys were young adults with a median age of 6 years (range, 5-9). The other 16 monkeys were older adults with a median age of 14 years (range, 10-17). Puberty occurs at 2-3 years of age, and the average maximum lifespan in captivity is $\sim 21$ years of age (Brady, 2000).

During assessments of cognitive performance, monkeys were housed and tested individually in wire-mesh cages $(60 \times 60 \times 90 \mathrm{~cm})$ that provided visual, auditory, and olfactory, but not direct physical contact, between familiar animals. At all other times, monkeys were socially housed in cages $(1.2 \times 1.2 \times 1.8 \mathrm{~m})$ that contained three or four familiar females of the same age. All cages were located in climate-controlled rooms with a $12 \mathrm{hr}$ light/dark cycle. Procedures complied with National Institutes of Health policies on the care and use of animals and were approved by the Stanford University animal care committee.

Cognitive tests. The apparatus used for the cognitive tests was a clear Plexiglas box $(8 \times 8 \times 8 \mathrm{~cm})$ with one open side that contained a marshmallow food treat (Lyons et al., 2000a). The box was mounted on a tray and secured to the front of each monkey's cage. Initially, each monkey was easily trained to retrieve food treats from the clear baited box. Thereafter, each monkey was administered a series of four five-trial sessions each day for 14 consecutive days. Each trial was terminated when the treat was retrieved, with a $20 \mathrm{sec}$ delay between trials in each session and a 30 min delay between each of the four daily five-trial sessions.

For the first two daily five-trial sessions, the box opening was oriented in the same direction (i.e., toward the left on even-numbered days and toward the right on odd-numbered days). Each monkey was required to learn and remember the initial box-opening orientation. On the second two daily five-trial sessions, the box opening was oriented opposite to that presented for the first two sessions. Each monkey was now required to inhibit the previously rewarded reaching response and remember the new orientation. After completion of the four daily sessions, all monkeys were fed unrestricted amounts of commercial monkey chow with fresh fruit and vegetable supplements. The following morning, $1 \mathrm{hr}$ before testing, all uneaten food was removed. Each monkey was tested at the same time of day, between 9:00 A.M. and 12:00 P.M.

Stress tests and hormone measures. Three months after the cognitive tests, plasma cortisol and adrenocorticotropic hormone (ACTH) responses evoked by a standardized test were measured in the squirrel monkey nonbreeding season. At this point, circannual rhythms in adrenal and gonadal steroid hormones in squirrel monkeys are at their nadir (Schiml et al., 1999). Cage mates were restrained adjacent to one another for $30 \mathrm{~min}$ in separate primate chairs, like those first described by Carmichael and MacLean (1961). Chair restraint provides slight room for movement and ample ventilation and does not induce pain or inflict tissue damage. Blood samples for cortisol and ACTH determinations were collected after the restraint-stress session at 0,30 , and 60 min intervals from monkeys socially housed in their own home cage.

To test for age-related differences in sensitivity to glucocorticoid negative-feedback regulation of the acute stress response, $7 \mathrm{~d}$ after the initial stress test, all monkeys were administered a second stress test preceded by an intramuscular (IM) injection of hydrocortisone sodium succinate $(20 \mathrm{mg} / \mathrm{kg})$. This dose of hydrocortisone is known to suppress squirrel monkey plasma levels of ACTH (Lyons et al., 2000b). Hydrocortisone was administered $60 \mathrm{~min}$ before the second $30 \mathrm{~min}$ restraint-stress test. Sixty minutes before the first stress test, an IM injection of saline was administered to control for injection pretreatment effects. Seven days before and $7 \mathrm{~d}$ after the two successive restraint-stress tests, additional blood samples were collected in undisturbed home cage conditions for baseline hormone determinations.

Blood samples were obtained, as described previously (Lyons et al., 1999), between 1:30 and 2:30 P.M. to control for known circadian effects. All samples were transferred to chilled tubes on ice, centrifuged at $4^{\circ} \mathrm{C}$, and plasma aliquots were stored at $-80^{\circ} \mathrm{C}$. Plasma cortisol and ACTH levels were measured in duplicate using established radioimmunoassays (Lyons et al., 1999, 2000b). The cortisol assay does not distinguish between cortisol and exogenous hydrocortisone and, therefore, plasma levels of ACTH served as our primary index of HPA-axis stress reactivity and feedback regulation. Complete sample subsets from each test condition and age class were included in every hormone assay. Intra- and inter-assay coefficients of variation were $1.4 \%$ and $6.4 \%$ for cortisol and $5.2 \%$ and $5.5 \%$ for ACTH, respectively.

Brain image acquisition and analysis. Three months after the restraintstress tests, brain images were acquired on a General Electric (Milwaukee, WI) Signa 3.0 T system with protocols developed specifically for squirrel monkeys (Lyons et al., 2001). All monkeys were scanned under anesthesia induced by a subcutaneous injection of $20 \mathrm{mg} / \mathrm{kg}$ ketamine hydrochloride, $4 \mathrm{mg} / \mathrm{kg}$ xylazine hydrochloride, and $0.04 \mathrm{mg} / \mathrm{kg}$ atropine sulfate. Body temperatures were maintained within the normal range using a cushioned heat pad. Ear plugs provided protection from noises generated by the scanner.

The first scan was acquired in the sagittal plane with a twodimensional sequential spoiled gradient pulse sequence. This initial localizer scan was used to standardize head tilt and rotation by assuring that two external markers (vitamin E capsules in the meatus of each ear) were aligned in the coronal and axial planes. The head was repositioned as required, and another sagittal localizer scan was performed. Head pitch was standardized against a midsagittal image, and the final scan used for volumetric analysis was acquired in the coronal plane with a threedimensional inversion recovery-prepared fast-spoiled gradient pulse sequence: repetition time $=12 \mathrm{msec}$; echo time $=3 \mathrm{msec}$; inversion 

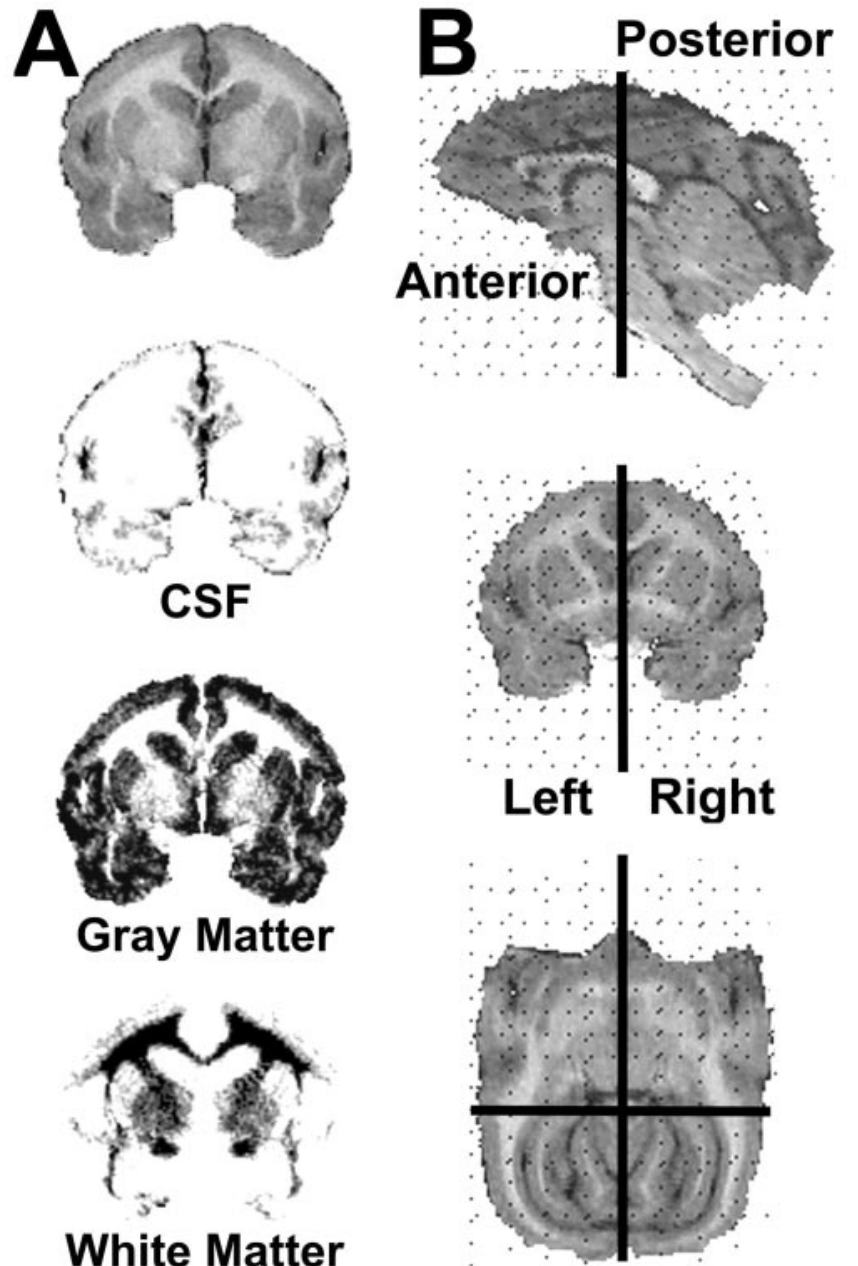

Figure 2. Brain image processing. $A$, Gray-scale image (top) from a representative monkey is segmented into CSF, gray, and white matter (top to bottom, respectively) with darker voxels depicting larger proportions of each tissue type. $B$, Multiplanar views of a gray-scale image show the stereotaxic Talaraich-like grid (dotted lines) used for parcellation of the brain image stack into four three-dimensional quadrants (solid lines).

time $=300$ msec; flip angle, 15; number of excitations, 4; matrix, $256 \times$ 256; field of view, $8 \mathrm{~cm}$; voxel size $=0.31 \times 0.31 \times 1.00 \mathrm{~mm}$; slice thickness, $1 \mathrm{~mm}$; gap, $0 \mathrm{~mm}$; total scan time, $17 \mathrm{~min}$. Inversion recoveryprepared protocols provide the maximum gray versus white matter contrast currently achievable with MRI (Bartzokis et al., 2001).

Standardized semiautomated image-processing protocols were performed as described for human subjects with the program BrainImage v5.27 on an Apple Macintosh G4 computer (Apple Computers, Cupertino, CA) (Patwardhan et al., 2001). Briefly, image processing included the removal of all nonbrain tissue, correction of equipment-related artifacts, segmentation into gray, white, and CSF components, positional normalization, and parcellation into proportional Talaraich-like stereotaxic sectors. These procedures produced volumetric measures of gray, white, and CSF in four three-dimensional complementary brain quadrants (Fig. 2). Hippocampal volumes were also determined by hand drawing anatomical boundaries described previously for adult squirrel monkeys (Lyons et al., 2001) onto coronal brain image stacks. Intra-rater reliabilities expressed as intra-class correlations from fixed effects models were $>0.90$.

Data analysis. Reach attempt errors summed across days were examined for age and test session effects using ANOVA with repeated measures in the multivariate general linear hypothesis module of Systat (Richmond, CA). Age class was considered as a between-subjects factor,

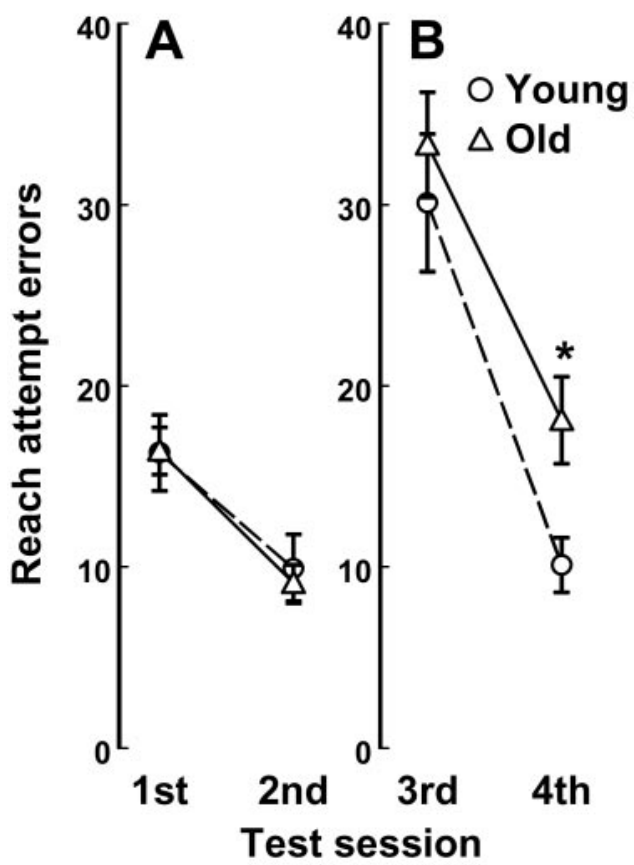

Figure 3. Age-related differences in cognitive performance. $A$, Reach attempt error rates did not differ with age before the box-opening orientation was reversed. $B$, After reversals, error rates increased, and older adults continued to make significantly more errors than young adults during the final test session. Data are presented as mean \pm SEM for 15 young and 16 older adults; ${ }^{*} p<0.01$.

and test session was the repeated measures factor. The Geisser-Greenhouse correction was used to adjust for multiple comparisons across all repeated measures factors. Similar procedures were used to assess age class and hydrocortisone pretreatment effects for poststress measures of cortisol and ACTH and age class differences in regional brain measures. Age effects were also examined using linear least squares regression with age as a continuous variable. Pearson correlations and partial correlations were used to assess the relationships between age, cognitive errors, stress hormone levels, and in vivo brain volumes. All statistics were evaluated with two-tailed probabilities $(p<0.05)$.

\section{Results}

Learning and memory was initially assessed with the box opening that was consistently presented toward either the left or right. The marshmallow treat was always retrieved from the one open side of the box, but successful retrievals were often preceded by reach attempt errors toward the incorrect side (Fig. 3A). Significantly fewer errors were observed across the first and second test sessions $\left(F_{(1,30)}=127.10 ; p<0.001\right)$, and young and older adults did not differ in learning the initial box-opening orientation.

After the first and second test sessions, the box-opening orientation was reversed. Third session error rates increased significantly, relative to the first $\left(F_{(1,30)}=78.92 ; p<0.001\right)$ and second $\left(F_{(1,30)}=155.87 ; p<0.001\right)$ test sessions. Both young and older adults performed as if the box opening had not been reversed. But during the subsequent fourth test session, $30 \mathrm{~min}$ after the spatial reversal, older adults continued to make more reach attempt errors than young adults $\left(F_{(1,29)}=7.88 ; p=0.009\right)$ (Fig. $\left.3 B\right)$. Age effects were also discerned, with age considered a continuous variable $\left(F_{(1,29)}=6.42 ; p=0.017\right)$. Fourth session error rates were nearly identical to second session error rates in young adults (Fig. 3B), whereas fourth session error rates were significantly greater than second session error rates in older adults $\left(F_{(1,15)}=\right.$ 29.82; $p<0.001)$. Fourth-minus-second session error rates com- 


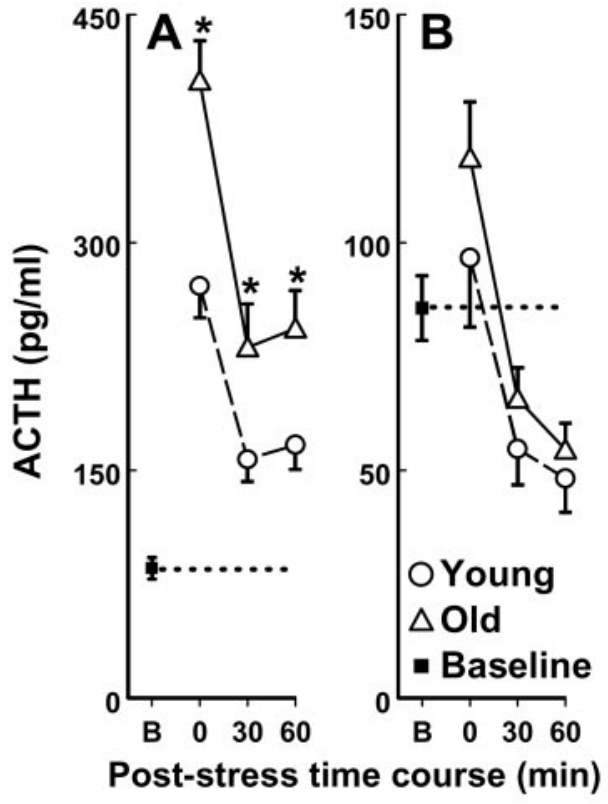

Figure 4. Age-related differences in stress reactivity and glucocorticoid feedback. A, Poststress levels of ACTH were greater in old than young adults after pretreatment with saline. $B_{\text {, }}$ After hydrocortisone, poststress levels of ACTH were equal to or less than baseline levels in young adults, but older adults failed to suppress below baseline the initial ACTH stress response. Data are presented as mean \pm SEM for 15 young and 16 older adults; ${ }^{*} p<0.01$.

puted for each monkey served as an index of impaired response inhibition that correlated significantly with increasing age $(r=$ 0.59 ; $\mathrm{df}=29 ; p<0.001)$. Older adults more often failed to inhibit the initial learned response after spatial reversals that required learning the reversed box-opening orientation.

Three months after the cognitive tests, stress reactivity and regulation of the HPA axis were examined. Before the study, all monkeys were trained to quickly enter a small holding cage to facilitate the rapid collection of samples for cortisol and ACTH determinations. Most samples (91\%) were collected within 3 min (median latency to sample collection, $110 \mathrm{sec}$; range, 25-354 sec), and all but three samples $(<2 \%)$ were collected within $4 \mathrm{~min}$. In keeping with evidence that plasma cortisol and $\mathrm{ACTH}$ in squirrel monkeys measured within these time limits do not reflect disturbance effects from the sampling procedure per se, sample collection latencies explained $1 \%$ of the variance in cortisol and ACTH.

Neither cortisol nor ACTH levels at baseline differed in young and older adults. However, monkeys with greater baseline levels responded with greater postrestraint levels of cortisol $(r=0.53$; $\mathrm{df}=29 ; p=0.002)$ and postrestraint levels of ACTH $(r=0.36$; $\mathrm{df}=29 ; p=0.047)$. Postrestraint measures were therefore analyzed with baseline levels that were considered a covariate to control for baseline variation.

Significant main effects of age class $\left(F_{(1,28)}=23.32 ; p<0.001\right)$ and time course $\left(F_{(2,56)}=14.01 ; p<0.001\right)$ were evident in postrestraint levels of ACTH when monkeys were pretreated with saline. Postrestraint levels of ACTH were greater than baseline but declined over time, and older adults at all time points responded with greater postrestraint levels of ACTH relative to young adults. These same age effects were likewise discerned without controlling for baseline as a covariate (Fig. 4A), and regardless of whether age was considered a continuous or categorical variable.

As expected, hydrocortisone pretreatment robustly sup-
Table 1. Correlations between age, cognitive errors, neuroendocrine measures, and anterior bilateral white matter volumes in 31 adult female squirrel monkeys

\begin{tabular}{lllll}
\hline & Age & $\begin{array}{l}\text { Cognitive } \\
\text { errors }\end{array}$ & $\begin{array}{l}\text { Stress } \\
\text { response }\end{array}$ & $\begin{array}{l}\text { Impaired } \\
\text { glucocorticoid } \\
\text { feedback }\end{array}$ \\
\hline Cognitive errors & $0.59^{* *}$ & & & \\
Stress response & $0.37^{*}$ & $0.53^{* *}$ & & \\
Impaired glucocorticoid feedback & 0.05 & 0.20 & $0.67^{* *}$ & \\
White matter volume & $0.43^{*}$ & $0.46^{*}$ & 0.28 & 0.15 \\
\hline${ }^{*}<<0.05 ; *{ }^{*}<0.01$. & & & &
\end{tabular}

pressed stress levels of ACTH (Fig. $4 B$ ) relative to pretreatment with saline (Fig. 4A). Postrestraint levels of ACTH after hydrocortisone analyzed without baseline variation controlled as a covariate did not differ significantly with age (Fig. $4 B$ ). Nevertheless, the age main effect was statistically significant when baseline was included as a covariate, regardless of whether age was considered as a continuous $\left(F_{(1,28)}=5.35 ; p=0.028\right)$ or categorical $\left(F_{(1,28)}=7.91 ; p=0.009\right)$ variable. Postrestraint levels of ACTH after hydrocortisone were equal to or less than baseline in young adults, but older adults failed to suppress below baseline the initial ACTH stress response (Fig. $4 B$ ). This modest difference in ACTH after hydrocortisone cannot be attributed to significant age differences in the overall size of the glucocorticoid feedback signal achieved by administration of hydrocortisone. Plasma levels of glucocorticoid (cortisol and hydrocortisone combined) did not differ with age, whether age was analyzed as a continuous or categorical variable.

Stress reactivity determined from each monkey's mean plasma level of ACTH after pretreatment with saline correlated with age $(r=0.37 ; \mathrm{df}=29 ; p=0.041)$ and diminished glucocorticoid feedback determined from each monkey's mean ACTH level after pretreatment with hydrocortisone $(r=0.67$; $\mathrm{df}=29$; $p<0.001$ ). The partial correlation between stress reactivity and diminished glucocorticoid feedback sensitivity was also significant after controlling for age-related variation $(r=0.69 ; \mathrm{df}=28$; $p<0.001)$. As summarized in Table 1 , stress reactivity $(r=0.53$; $\mathrm{df}=29 ; p=0.002)$ but not diminished glucocorticoid feedback $(r=0.20 ; \mathrm{df}=29 ; p=0.287)$ was likewise associated with impaired cognitive response inhibition determined from each monkey's fourth-minus-second session errors. The correlation between cognitive performance and stress reactivity was significantly greater than that between cognitive performance and diminished glucocorticoid feedback $(t=2.66 ; \mathrm{df}=28 ; p=$ $0.013)$.

Three months after the neuroendocrine tests, unilateral volumes of gray and white matter in the anterior and posterior brain sectors were measured and analyzed for age-related differences with brain side considered a within-subjects factor. Bonferroni corrections were applied to control for comparisons across multiple brain regions. Each test statistic from the ANOVAs for each of the four measures of interest was assessed at $p<0.0125$. Significant main effects of brain side were evident with right volumes greater than left volumes for the white but not gray matter measures (Table 2). The only significant age effect was found in anterior white matter (age class main effect, $F_{(1,29)}=9.61 ; p=$ $0.004)$. Anterior volumes of white matter measured separately on the left $\left(F_{(1,29)}=9.85 ; p=0.004\right)$ and right $\left(F_{(1,29)}=8.12 ; p=\right.$ 0.008 ) sides were $17-19 \%$ larger in older adults than in young adult monkeys. Age effects were not significant for gray matter measures or hippocampal volumes analyzed as either unilateral or bilateral (left and right combined) measures with cranial volume controlled as a statistical covariate. 
Table 2. Asymmetries in gray and white matter volumes in 31 adult female squirrel monkeys

\begin{tabular}{lll}
\hline & Left & Right \\
\hline Anterior & & \\
Gray & $2939 \pm 60$ & $2973 \pm 62$ \\
White & $1481 \pm 48$ & $1574 \pm 48^{*}$ \\
Posterior & & \\
Gray & $3371 \pm 101$ & $3412 \pm 105$ \\
White & $1843 \pm 64$ & $1940 \pm 65^{*}$ \\
\hline
\end{tabular}

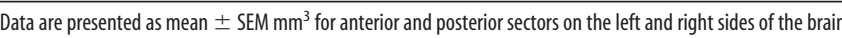
$\left({ }^{*} p<0.01\right)$.

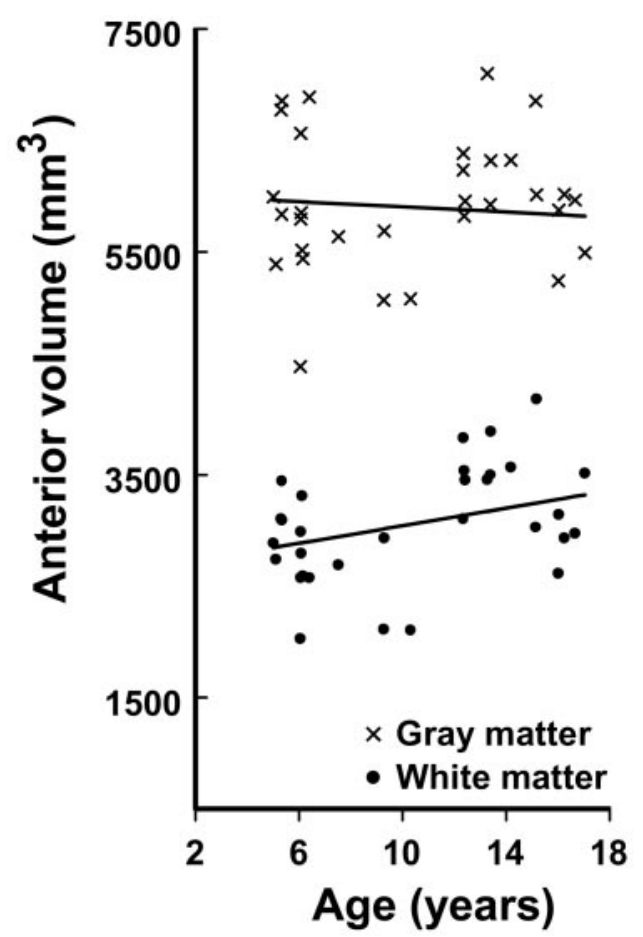

Figure 5. Regression of age on anterior bilateral gray and white matter volumes in 31 adult female squirrel monkeys.

To examine more closely the differential effects of age on anterior gray and white matter, regression analyses were subsequently performed for bilateral measures of gray and white matter with cranial volume as a covariate (Fig. 5). White matter volume increased with age $\left(F_{(1,28)}=6.55 ; p=0.016\right)$, whereas modest reductions in gray matter volume were not statistically significant $\left(F_{(1,28)}=1.28 ; p=0.268\right)$. Regression coefficients from these analyses showed that the linear increase per year for white matter volume $\left(39.4 \mathrm{~mm}^{3}\right)$ was threefold greater than the linear decrease in anterior gray matter $\left(-11.7 \mathrm{~mm}^{3}\right)$. Because a small proportion of brain is comprised of anterior white matter (Table 2), the overall change in total brain tissue volume was not significant $\left(F_{(1,28)}=0.038 ; p=0.848\right)$.

Impaired cognitive response inhibition, determined from fourth-minus-second session error rates, correlated with larger anterior white matter volumes on the left $(r=0.50$; df $=29 ; p=$ $0.004)$ and right $(r=0.39 ; \mathrm{df}=29 ; p=0.031)$ brain sides. Partial correlations controlling for age, reactivity to stress, anterior gray matter, and CSF volumes determined that left $(r=0.39$; $\mathrm{df}=25$; $p=0.043)$ but not right $(r=0.11 ; \mathrm{df}=25 ; p=0.578)$ anterior white matter contributed uniquely to impaired cognitive response inhibition. White matter volume in anterior brain regions did not correlate significantly with stress reactivity nor with diminished glucocorticoid feedback, regardless of whether white matter measures were analyzed as separate unilateral volumes or combined as a bilateral measure (Table 1).

\section{Discussion}

Neither cortisol nor ACTH levels at baseline differed with age during midlife adulthood, but older adult monkeys responded to stress with larger increases in HPA hormones relative to young adults. Stress reactivity was mediated in part by modest reductions in glucocorticoid feedback sensitivity determined by administration of exogenous hydrocortisone. Stress reactivity also correlated with age-related differences in cognitive control. Cognitive performance did not differ with age during the first or second test sessions, but older adults more often failed to inhibit the initial learned response after subsequent spatial reversals. Impaired cognitive response inhibition correlated with the expansion of white matter volume in anterior but not posterior brain regions statistically controlling for age effects, neuroendocrine responses to psychological stress, CSF, and gray matter volumes. These results indicate that instead of enhancing cognitive control during midlife adulthood, white matter volume expansion contributes to aspects of cognitive decline.

Neurobiological studies of stress and cognitive decline during aging tend to focus on the hippocampus (Lupien and Lepage, 2001; Miller and O'Callaghan, 2003). Our findings reflect a different aspect of cognition, because prefrontal lesions (Diamond et al., 1989; Jentsch et al., 1997; Wallis et al., 2001) and not hippocampal ablations (Diamond et al., 1989) impair response inhibition on tests like those administered here to healthy nonlesioned squirrel monkeys. Moreover, we failed to find age-related differences in monkey hippocampal volumes determined in vivo by MRI. Hippocampal atrophy gradually emerges during the mid-fifties in healthy humans (Raz et al., 2004) and may occur earlier in men compared with healthy middle-age women (Pruessner et al., 2001). All but 5 of the 31 monkeys in our study were younger than the equivalent of 55 years in human development, and all of the monkeys were females. The absence of hippocampal atrophy in monkeys therefore agrees with human studies and cannot be generalized to adult males or elderly female squirrel monkeys.

Age-related differences in hippocampal-dependent memory were not evident during the prereversal tests, but older middleage adult female squirrel monkeys more often failed to inhibit the initial learned response after spatial reversals designed to assess prefrontal-dependent cognitive functions. Similar studies of adult rhesus monkeys indicate that spatial but not object reversal learning is an especially sensitive marker of prefrontal cognitive decline (Bartus et al., 1979; Lai et al., 1995). Human abilities that likewise rely on prefrontal-dependent cognitive control, spatial orientation, inhibition, and reasoning also show approximately linear declines throughout adult midlife development (Baltes et al., 1999; McArdle et al., 2002; De Luca et al., 2003). In humans and monkeys, stress hormones impair prefrontal-dependent cognitive control (Young et al., 1999; Lyons et al., 2000a), and monkey cognitive response inhibition impairments after spatial reversals correlate with increased stress reactivity as determined by HPA hormones.

Although squirrel monkey HPA-axis physiology differs from humans because of mutations in the glucocorticoid receptor and related chaperones that result in glucocorticoid resistance (Chrousos et al., 1982; Patel et al., 2000a; Scammell et al., 2001), the high cortisol set-point in monkeys counteracts end-organ 
resistance (Cassorla et al., 1982; Moore et al., 1993), and squirrel monkeys demonstrate otherwise normal regulation of the HPA hormone stress response. Corticotropic-releasing factor stimulates secretion of pituitary ACTH (Lyons et al., 2000b), which stimulates secretion of cortisol from the squirrel monkey adrenal cortex (Lyons et al., 1995). Exogenous or endogenous stress levels of cortisol, in turn, suppress the secretion of squirrel monkey ACTH (Lyons et al., 1999, 2000a,b).

Relative to young adult female squirrel monkeys, older middle-age adult females exhibit greater stress hormone responses and modest reductions in sensitivity to glucocorticoid feedback. These findings agree with evidence that increased stress reactivity is mediated in part by diminished glucocorticoid feedback sensitivity with aging in adult rhesus monkeys, baboons, and healthy humans (Sapolsky and Altmann, 1991; Gust et al., 2000; Wilkinson et al., 2001). Diminished sensitivity to glucocorticoid feedback during aging results from decreased glucocorticoid receptor densities (Sapolsky, 1999) and age-related impairments in glucocorticoid receptor signaling (Murphy et al., 2002) in the adult rodent hippocampus. Glucocorticoid receptors are expressed throughout the hippocampus and neocortex in squirrel monkeys (Patel et al., 2000b) and rhesus macaques (Sanchez et al., 2000), but little is known about age-related changes in glucocorticoid receptors in primates.

Glucocorticoid receptors also reside in myelin-producing oligodendrocytes (Vielkind et al., 1990), and glucocorticoid receptor signaling stimulates myelination of axons in vitro (Chan et al., 1998; Afshari et al., 2002). However, white matter volumes in squirrel monkeys are not correlated with glucocorticoid levels at baseline or after an acute restraint stress. White matter volumes in anterior but not posterior brain regions on the left and right sides are significantly larger in older middle-age adult monkeys compared with young adults. This white matter volume expansion does not result in an overall change in brain size, because a small proportion of brain is comprised of anterior white matter. Gray matter volumes gradually decline, but the decrease is threefold less than the increase discerned in anterior white matter. Therefore, it seems unlikely that white matter growth during midlife adulthood is caused by a shift in gray-white T1 contrast with age. In human brain imaging, $\mathrm{T} 1$ is stable throughout adult midlife development compared with the changes observed in childhood, adolescence, and late adulthood (Cho et al., 1997; Steen and Schroeder, 2003). Moreover, postmortem studies of humans and monkeys suggest that in midlife adulthood, subtle cell shrinkage and modest pruning of synaptic connectivity in gray matter is accompanied by continued myelination of axon connections in anterior brain regions (Yakovlev and Lecours, 1967; Huttenlocher and Dabholkar, 1997; Peters and Sethares, 2002; Uylings and de Brabander, 2002; Duan et al., 2003; Peters and Sethares, 2003).

Myelination increases nerve conduction velocities (Baumann and Pham-Dinh, 2001) and facilitates synchronous firing of neurons by reducing the effects of travel distance variability in distributed brain circuits (Waxman, 1997; Lang and Rosenbluth, 2003; Salami et al., 2003). Frontal and temporal lobe white matter growth has therefore been viewed as enhancing neuropsychological functions that rely on distributed networks in humans (Greenwood, 2000; Bartzokis et al., 2001; Mulhern et al., 2001; Giedd, 2003). However, instead of enhancing cognitive control during midlife adulthood in monkeys, our results indicate that white matter volume expansion in anterior brain regions corresponds with impaired cognitive control of a simple learned response. These results are in keeping with postmortem studies that show how continued myelination coincides with increased accumulation of myelin decompaction defects that correlate in rhesus monkeys with aspects of cognitive decline (Peters et al., 2001; Peters and Sethares, 2002, 2003). Increased stress reactivity and diminished regulation of the HPA axis are additional aspects of aging that conflict with the view that white matter volume expansion improves distributed brain functions. However, white matter volumes in monkeys are not correlated with measures of stress reactivity or glucocorticoid feedback.

The extent to which data from monkeys can be generalized to aspects of aging in humans is limited in several respects. Frontal and temporal lobe white matter volumes in humans increase throughout childhood, adolescence, and early and midlife adulthood and then decline after 50 years of age (Giedd et al., 1999; Courchesne et al., 2000; Bartzokis et al., 2001; Jernigan and Fennema-Notestine, 2004). Many more measures from monkeys are needed to precisely define lifespan trajectories of white matter growth and subsequent decline for comparative studies with humans. White matter hyperintensities discerned by MRI on T2weighted images (Gunning-Dixon and Raz, 2000) are a prominent aspect of human brain aging that has not been examined in monkeys. Of particular interest is a recent report that the development of perseverative behavior in midlife adulthood correlates with increased white matter hyperintensities in the human frontal lobe (Gunning-Dixon and Raz, 2003). Gender comparisons also warrant attention in light of sex differences in white matter trajectories (Bartzokis, 2004) and human hemispheric asymmetries (Allen et al., 2003). In monkeys, larger right than left volumes of white but not gray matter resemble asymmetries in humans (Allen et al., 2003; Raz et al., 2004), with gender differences generally more prominent in white than gray matter tissue (Allen et al., 2003; Szeszko et al., 2003). Aging likewise appears to increase HPA-axis stress reactivity and diminish glucocorticoid feedback sensitivity to a greater extent in women than in men (Seeman et al., 2001).

Apart from these issues, our study highlights an additional major concern. Instead of revealing favorable forms of myelination in midlife adulthood, white matter volume expansion determined by neuroimaging may actually reflect increased accumulation of myelin decompaction defects that correlate with cognitive decline. The hypothesis that white matter growth represents myelin decompaction agrees with diffusion tensor MRI evidence that interstitial or intracellular fluid accumulates linearly with age in adult human frontal lobe white matter tissue determined by microstructural changes in water proton diffusivity (Abe et al., 2002; Moseley, 2002; Pfefferbaum and Sullivan, 2003). Cellular and molecular research combined with in vivo brain imaging is needed to determine the basis of white matter growth in adults, elucidate its functions during lifespan development, and provide potential new targets for therapies aimed at maintaining in humans cognitive vitality with aging.

\section{References}

Abe O, Aoki S, Hayashi N, Yamada H, Kunimatsu A, Mori H, Yoshikawa T, Okubo T, Ohtomo K (2002) Normal aging in the central nervous system: quantitative MR diffusion-tensor analysis. Neurobiol Aging 23:433-441.

Afshari FS, Chu AK, Sato-Bigbee C (2002) Recovery of adult oligodendrocytes is preceded by a "lag period" accompanied by upregulation of transcription factors expressed in developing young cells. J Neurosci Res 67:174-184.

Allen JS, Damasio H, Grabowski TJ, Bruss J, Zhang W (2003) Sexual dimorphism and asymmetries in the gray-white composition of the human cerebrum. NeuroImage 18:880-894. 
Baltes PB, Staudinger UM, Lindenberger U (1999) Lifespan psychology: theory and application to intellectual functioning. Annu Rev Psychol 50:471-507.

Bartus RT, Dean RL, Fleming DL (1979) Aging in the rhesus monkey: effects on visual discrimination learning and reversal learning. J Gerontol 34:209-219.

Bartzokis G (2004) Quadratic trajectories of brain myelin content: unifying construct for neuropsychiatric disorders. Neurobiol Aging 25:49-62.

Bartzokis G, Beckson M, Lu PH, Nuechterlein KH, Edwards N, Mintz J (2001) Age-related changes in frontal and temporal lobe volumes in men: a magnetic resonance imaging study. Arch Gen Psychiatry 58:461-465.

Baumann N, Pham-Dinh D (2001) Biology of oligodendrocyte and myelin in the mammalian central nervous system. Physiol Rev 81:871-927.

Brady AG (2000) Res techniques for the squirrel monkey (Saimiri). ILAR J $41: 10-18$.

Carmichael M, Maclean PD (1961) Use of squirrel monkey for brain research, with description of restraining chair. Electroencephalogr Clin Neurophysiol 13:128-129.

Cassorla FG, Albertson BD, Chrousos GP, Booth JD, Renquist D, Lipsett MB, Loriaux DL (1982) The mechanism of hypercortisolemia in the squirrel monkey. Endocrinology 111:448-451.

Chan JR, Phillips Jr LJ, Glaser M (1998) Glucocorticoids and progestins signal the initiation and enhance the rate of myelin formation. Proc Natl Acad Sci USA 95:10459-10464.

Cho S, Jones D, Reddick WE, Ogg RJ, Steen RG (1997) Establishing norms for age-related changes in proton T1 of human brain tissue in vivo. Magn Reson Imaging 15:1133-1143.

Chrousos GP, Renquist D, Brandon D, Eil C, Pugeat M, Vigersky R, Cutler Jr GB, Loriaux DL, Lipsett MB (1982) Glucocorticoid hormone resistance during primate evolution: receptor-mediated mechanisms. Proc Natl Acad Sci USA 79:2036-2040.

Courchesne E, Chisum HJ, Townsend J, Cowles A, Covington J, Egaas B, Harwood M, Hinds S, Press GA (2000) Normal brain development and aging: quantitative analysis at in vivo MR imaging in healthy volunteers. Radiology 216:672-682.

De Luca CR, Wood SJ, Anderson V, Buchanan JA, Proffitt TM, Mahony K, Pantelis C (2003) Normative data from the CANTAB. I. Development of executive function over the lifespan. J Clin Exp Neuropsychol $25: 242-254$

Diamond A, Zola-Morgan S, Squire LR (1989) Successful performance by monkeys with lesions of the hippocampal formation on $\mathrm{AB}$ and object retrieval, two tasks that mark developmental changes in human infants. Behav Neurosci 103:526-537.

Duan H, Wearne SL, Rocher AB, Macedo A, Morrison JH, Hof PR (2003) Age-related dendritic and spine changes in corticocortically projecting neurons in macaque monkeys. Cereb Cortex 13:950-961.

Eichenbaum H (2000) A cortical-hippocampal system for declarative memory. Nat Rev Neurosci 1:41-50.

Giedd JN (2003) The anatomy of mentalization: a view from developmental neuroimaging. Bull Menninger Clin 67:132-142.

Giedd JN, Blumenthal J, Jeffries NO, Castellanos FX, Liu H, Zijdenbos A, Paus T, Evans AC, Rapoport JL (1999) Brain development during childhood and adolescence: a longitudinal MRI study. Nat Neurosci 2:861-863.

Greenwood PM (2000) The frontal aging hypothesis evaluated. J Int Neuropsychol Soc 6:705-726.

Gunning-Dixon FM, Raz N (2000) The cognitive correlates of white matter abnormalities in normal aging: a quantitative review. Neuropsychology 14:224-232.

Gunning-Dixon FM, Raz N (2003) Neuroanatomical correlates of selected executive functions in middle-aged and older adults: a prospective MRI study. Neuropsychologia 41:1929-1941.

Gust DA, Wilson ME, Stocker T, Conrad S, Plotsky PM, Gordon TP (2000) Activity of the hypothalamic-pituitary-adrenal axis is altered by aging and exposure to social stress in female rhesus monkeys. J Clin Endocrinol Metab 85:2556-2563.

Herman JP, Figueiredo H, Mueller NK, Ulrich-Lai Y, Ostrander MM, Choi DC, Cullinan WE (2003) Central mechanisms of stress integration: hierarchical circuitry controlling hypothalamo-pituitary-adrenocortical responsiveness. Front Neuroendocrinol 24:151-180.

Huttenlocher PR, Dabholkar AS (1997) Regional differences in synaptogenesis in human cerebral cortex. J Comp Neurol 387:167-178.
Jentsch JD, Redmond Jr DE, Elsworth JD, Taylor JR, Youngren KD, Roth RH (1997) Enduring cognitive deficits and cortical dopamine dysfunction in monkeys after long-term administration of phencyclidine. Science 277:953-955.

Jernigan TL, Fennema-Notestine C (2004) White matter mapping is needed. Neurobiol Aging 25:37-39.

Kumar A, Cook IA (2002) White matter injury, neural connectivity and the pathophysiology of psychiatric disorders. Dev Neurosci 24:255-261.

Lai ZC, Moss MB, Killiany RJ, Rosene DL, Herndon JG (1995) Executive system dysfunction in the aged monkey: spatial and object reversal learning. Neurobiol Aging 16:947-954.

Lang EJ, Rosenbluth J (2003) Role of myelination in the development of a uniform olivocerebellar conduction time. J Neurophysiol 89:2259-2270.

Lupien SJ, Lepage M (2001) Stress, memory, and the hippocampus: can't live with it, can't live without it. Behav Brain Res 127:137-158.

Lupien SJ, de Leon M, de Santi S, Convit A, Tarshish C, Nair NP, Thakur M, McEwen BS, Hauger RL, Meaney MJ (1998) Cortisol levels during human aging predict hippocampal atrophy and memory deficits. Nat Neurosci 1:69-73.

Lyons DM, Ha CM, Levine S (1995) Social effects and circadian rhythms in squirrel monkey pituitary-adrenal activity. Horm Behav 29:177-190.

Lyons DM, Wang OJ, Lindley SE, Levine S, Kalin NH, Schatzberg AF (1999) Separation induced changes in squirrel monkey hypothalamic-pituitaryadrenal physiology resemble aspects of hypercortisolism in humans. Psychoneuroendocrinology 24:131-142.

Lyons DM, Lopez JM, Yang C, Schatzberg AF (2000a) Stress-level cortisol treatment impairs inhibitory control of behavior in monkeys. J Neurosci 20:7816-7821.

Lyons DM, Yang C, Mobley BW, Nickerson JT, Schatzberg AF (2000b) Early environmental regulation of glucocorticoid feedback sensitivity in young adult monkeys. J Neuroendocrinol 12:723-728.

Lyons DM, Yang C, Sawyer-Glover AM, Moseley ME, Schatzberg AF (2001) Early life stress and inherited variation in monkey hippocampal volumes. Arch Gen Psychiatry 58:1145-1151.

McArdle JJ, Ferrer-Caja E, Hamagami F, Woodcock RW (2002) Comparative longitudinal structural analyses of the growth and decline of multiple intellectual abilities over the life span. Dev Psychol 38:115-142.

McEwen BS (1999) Stress and the aging hippocampus. Front Neuroendocrinol 20:49-70.

Mesulam MM (1998) From sensation to cognition. Brain 121:1013-1052.

Miller DB, O'Callaghan JP (2003) Effects of aging and stress on hippocampal structure and function. Metabolism 52:17-21.

Moore CC, Mellon SH, Murai J, Siiteri PK, Miller WL (1993) Structure and function of the hepatic form of 11 beta-hydroxysteroid dehydrogenase in the squirrel monkey, an animal model of glucocorticoid resistance. Endocrinology 133:368-375.

Moseley M (2002) Diffusion tensor imaging and aging-a review. NMR Biomed 15:553-560.

Mulhern RK, Palmer SL, Reddick WE, Glass JO, Kun LE, Taylor J, Langston J, Gajjar A (2001) Risks of young age for selected neurocognitive deficits in medulloblastoma are associated with white matter loss. J Clin Oncol 19:472-479.

Murphy EK, Spencer RL, Sipe KJ, Herman JP (2002) Decrements in nuclear glucocorticoid receptor (GR) protein levels and DNA binding in aged rat hippocampus. Endocrinology 143:1362-1370.

Patel PD, Lyons DM, Zhang Z, Ngo H, Schatzberg AF (2000a) Impaired transactivation of the glucocorticoid receptor cloned from the Guyanese squirrel monkey. J Steroid Biochem Mol Biol 72:115-123.

Patel PD, Lopez JF, Lyons DM, Burke S, Wallace M, Schatzberg AF (2000b) Glucocorticoid and mineralocorticoid receptor mRNA expression in squirrel monkey brain. J Psychiatr Res 34:383-392.

Patwardhan AJ, Eliez S, Warsofsky IS, Glover GH, White CD, Giedd JN, Peterson BS, Rojas DC, Reiss AL (2001) Effects of image orientation on the comparability of pediatric brain volumes using three-dimensional MR data. J Comput Assist Tomogr 25:452-457.

Peters A (1996) Age-related changes in oligodendrocytes in monkey cerebral cortex. J Comp Neurol 371:153-163.

Peters A, Sethares C (2002) Aging and the myelinated fibers in prefrontal cortex and corpus callosum of the monkey. J Comp Neurol 442:277-291.

Peters A, Sethares C (2003) Is there remyelination during aging of the primate central nervous system? J Comp Neurol 460:238-254.

Peters A, Rosene DL, Moss MB, Kemper TL, Abraham CR, Tigges J, Albert MS 
(1996) Neurobiological bases of age-related cognitive decline in the rhesus monkey. J Neuropathol Exp Neurol 55:861-874.

Peters A, Sethares C, Killiany RJ (2001) Effects of age on the thickness of myelin sheaths in monkey primary visual cortex. J Comp Neurol 435:241-248.

Pfefferbaum A, Sullivan EV (2003) Increased brain white matter diffusivity in normal adult aging: relationship to anisotropy and partial voluming. Magn Reson Med 49:953-961.

Preuss TM (1995) Do rats have prefrontal cortex? The Rose-Woolsey-Akert program reconsidered. J Cogn Neurosci 7:1-24.

Pruessner JC, Collins DL, Pruessner M, Evans AC (2001) Age and gender predict volume decline in the anterior and posterior hippocampus in early adulthood. J Neurosci 21:194-200.

Raz N, Gunning-Dixon F, Head D, Rodrigue KM, Williamson A, Acker JD (2004) Aging, sexual dimorphism, and hemispheric asymmetry of the cerebral cortex: replicability of regional differences in volume. Neurobiol Aging 25:377-396.

Salami M, Itami C, Tsumoto T, Kimura F (2003) Change of conduction velocity by regional myelination yields constant latency irrespective of distance between thalamus and cortex. Proc Natl Acad Sci USA 100:6174-6179.

Sanchez MM, Young LJ, Plotsky PM, Insel TR (2000) Distribution of corticosteroid receptors in the rhesus brain: relative absence of glucocorticoid receptors in the hippocampal formation. J Neurosci 20:4657-4668.

Sapolsky RM (1999) Glucocorticoids, stress, and their adverse neurological effects: relevance to aging. Exp Gerontol 34:721-732.

Sapolsky RM, Altmann J (1991) Incidence of hypercortisolism and dexamethasone resistance increases with age among wild baboons. Biol Psychiatry 30:1008-1016.

Scammell JG, Denny WB, Valentine DL, Smith DF (2001) Overexpression of the FK506-binding immunophilin FKBP51 is the common cause of glucocorticoid resistance in three New World primates. Gen Comp Endocrinol 124:152-165.

Schiml PA, Mendoza SP, Saltzman W, Lyons DM, Mason WA (1999) An- nual physiological changes in individually housed squirrel monkeys (Saimiri sciureus). Am J Primatol 47:93-103.

Seeman TE, Singer B, Wilkinson CW, McEwen B (2001) Gender differences in age-related changes in HPA axis reactivity. Psychoneuroendocrinology 26:225-240.

Steen RG, Schroeder J (2003) Age-related changes in the pediatric brain: proton $\mathrm{T} 1$ in healthy children and in children with sickle cell disease. Magn Reson Imaging 21:9-15.

Szeszko PR, Vogel J, Ashtari M, Malhotra AK, Bates J, Kane JM, Bilder RM, Frevert T, Lim K (2003) Sex differences in frontal lobe white matter microstructure: a DTI study. NeuroReport 14:2469-2473.

Uylings HB, de Brabander JM (2002) Neuronal changes in normal human aging and Alzheimer's disease. Brain Cogn 49:268-276.

Vielkind U, Walencewicz A, Levine JM, Bohn MC (1990) Type II glucocorticoid receptors are expressed in oligodendrocytes and astrocytes. J Neurosci Res 27:360-373.

Walker LC, Masters C, Beyreuther K, Price DL (1990) Amyloid in the brains of aged squirrel monkeys. Acta Neuropathol 80:381-387.

Wallis JD, Dias R, Robbins TW, Roberts AC (2001) Dissociable contributions of the orbitofrontal and lateral prefrontal cortex of the marmoset to performance on a detour reaching task. Eur J Neurosci 13:1797-1808.

Waxman SG (1997) Axon-glia interactions: building a smart nerve fiber. Curr Biol 7:R406-R410

Wilkinson CW, Petrie EC, Murray SR, Colasurdo EA, Raskind MA, Peskind ER (2001) Human glucocorticoid feedback inhibition is reduced in older individuals: evening study. J Clin Endocrinol Metab 86:545-550.

Yakovlev PI, Lecours A (1967) The myelogenetic cycles of regional maturation in the brain. In: Regional development of the brain in early life (Minkowski A, ed), pp 3-65. Oxford: Blackwell.

Young AH, Sahakian BJ, Robbins TW, Cowen PJ (1999) The effects of chronic administration of hydrocortisone on cognitive function in normal male volunteers. Psychopharmacology (Berl) 145:260-266. 